\title{
Emiratos Árabes Unidos en Oriente Medio. Antiislamismo, militarismo y estrategia de presión múltiple
}

\author{
The United Arab Emirates in the Middle East. \\ Anti-islamism, militarism, and multiple-pressure strategy
}

\author{
JAVIER GUIRADO \\ Georgia State University \\ IGNACIO GUTIÉRREZ DE TERÁN \\ Universidad Autónoma de Madrid
}

\section{Cómo citar/Citation}

Guirado, J. y Gutiérrez de Terán, I. (2021). Emiratos Árabes Unidos en Oriente Medio. Antiislamismo, militarismo y estrategia de presión múltiple. Revista Española de Ciencia Política, 56, 71-96. Doi: https://doi.org/10.21308/recp.56.03

\section{Resumen}

La política exterior de los Emiratos Árabes Unidos (EAU) ha experimentado un cambio sustancial desde el inicio de la denominada Primavera Árabe en 2011. Este artículo describe las características y razones principales de esta transformación, así como las posibles consecuencias de una proyección militar que rebasa los límites del golfo Árabe/Pérsico, hasta hace poco el principal ámbito de acción de la diplomacia emiratí. El enfoque toma en consideración, en primer lugar, la vinculación tradicional de EE. UU. con EAU y el cambio de percepción de las últimas Administraciones estadounidenses sobre la seguridad regional en el Golfo; la consagración del emir Mohammad ben Zayed como máximo dirigente de facto en el país y su alianza con el príncipe heredero saudí, Mohammed ben Salman; el ánimo de neutralizar la corriente democratizadora emanada de las revoluciones árabes y, a partir de ahí, la percepción emiratí de que el islam político, y en concreto la vertiente representada por los Hermanos Musulmanes, se estaba convirtiendo en el principal beneficiado de la erosión del régimen árabe tradicional. El artículo también propone que, a pesar de la convergencia con Arabia Saudí en numerosos escenarios, EAU ha mantenido una política exterior independiente basada en sus propios intereses, lo que ha reforzado su rol como principal contendiente en Oriente Medio.

Palabras clave: Emiratos Árabes Unidos, política exterior, Primavera Árabe, islam político, Golfo, Mohammed ben Zayed. 


\begin{abstract}
The United Arab Emirates' (UAE) foreign policy has experienced substantial changes since the beginning of the so-called Arab Spring in 2011. This paper describes the characteristics and main reasons of this transformation, as well as the possible consequences of a visible militaristic projection that goes beyond the limits of the Gulf region, the main scenario of Emirati diplomatic action until recently. Our framework takes into account, first of all, the traditional link between the United States and the UAE and how recent US administrations changed their perception about security in the Gulf; how Emir Mohammed bin Zayed has secured his grip over the country, and the relevance of his alliance with the Saudi Crown Prince Mohammed bin Salman; the aim of neutralizing the democratic stream that emerged from the Arab revolutions and, from there on, the Emirati perception about how political Islam and particularly the current best represented by the Muslim Brotherhood was becoming the main beneficiary of the erosion of the traditional Arab regime. Moreover, this paper proposes that despite the convergence with Saudi Arabia in a number of scenarios, the UAE has kept a foreign policy based on its own interests, which strengthened its increasing role as a regional main contender in the Middle East.
\end{abstract}

Keywords: United Arab Emirates, foreign policy, Arab Spring, political Islam, Arab/Persian Gulf, Mohammed bin Zayed.

\title{
INTRODUCCIÓN
}

Las revueltas de 2011 en los países árabes cambiaron el escenario político de la región. La contestación popular a regímenes y las protestas centradas en la corrupción promovieron fuerzas políticas a nivel regional entre las que el islamismo político gozó de gran auge. Este trabajo pretende demostrar cómo este escenario ha provocado que EAU potencie su presencia en la región defendiendo una agenda explícitamente antiislamista, en un marco que incluye factores como la progresiva retirada de Estados Unidos (EE. UU) de Oriente Medio, la apuesta de Catar por los partidos islamistas o la frecuente convergencia de intereses con Arabia Saudí, a pesar de que EAU ha desarrollado una política independiente del reino wahabí.

Para ello, el artículo propone un process tracing de la actuación de EAU dividido cronológicamente en tres partes. La primera parte estudia los orígenes de la doctrina exterior emiratí en relación con la independencia del imperio británico, Irán o la influencia de conflictos como la guerra del Golfo. La segunda parte analiza la progresiva militarización de la política exterior emiratí durante la última década y media en el marco de la Administración Obama, las relaciones con Rusia y China, la creciente hostilidad hacia el islam político y la expansión de la influencia de EAU a nivel económico y de soft power. El tercer apartado estudia la intervención y actitud de EAU en Bahréin, Siria, Libia, Yemen, Egipto, Catar, el Cuerno de África, Palestina y la normalización con Israel, demostrando a través de las convergencias y divergencias con Arabia Saudí que EAU ha desarrollado una política exterior independiente 
basada en sus propios incentivos a nivel económico y político y, particularmente entre 2016 y 2020, dentro de una firme alianza con la Administración Trump. El principal objetivo del artículo es demostrar que EAU se ha convertido en un actor de primer nivel en la región del Golfo, con una línea de actuación propia que sigue unas coordenadas no siempre ajustadas a los intereses principales de la gran potencia regional, Arabia Saudí.

\section{MARCO TEÓRICO, DISEÑO DE LA INVESTIGACIÓN Y METODOLOGÍA}

El trabajo se fundamenta en una metodología cualitativa basada en el process-tracing. Como señala Collier (2011), el process-tracing es una herramienta para extraer mecanismos causales a través de evidencias basadas en una secuencia de eventos, como forma de abrir la caja negra para explicar dichas actuaciones. Goertz y Mahoney señalan el enorme potencial del process-tracing para estudios de caso desde un enfoque cualitativo (2012: 87), mientras que Owen (1994), en su trabajo sobre paz entre democracias liberales, se centra en los mecanismos causales a través de varios casos históricos. Esta metodología permite mostrar las implicaciones de la hipótesis como una secuencia temporal observable. El objetivo de este estudio es explicar si la política exterior de EAU está determinada por el temor a la expansión del islam político en Oriente Medio. La hipótesis de partida es que, tras el ascenso de Mohammed bin Zayed (MbZ) como príncipe heredero, y sobre todo tras las revueltas en el mundo árabe de 2011, la federación ha entendido que el islamismo podía extenderse también en EAU y constituir una fuerza de oposición contra el liderazgo de la familia $\mathrm{Al}$ Nahyan. Por ello, en un juego de espejos entre política interior y exterior, EAU se ha centrado en intentar eliminar cualquier fuerza islamista en Oriente Medio siguiendo lo que hemos denominado doctrina $M b Z$. Tangencialmente a esta hipótesis, el trabajo también propone que la política exterior de EAU no está determinada por Arabia Saudí, en tanto que variable de control que se examina en cada caso, y que pese a ser aliado en numerosos escenarios ha participado en bandos opuestos en casos como el de Yemen.

La secuencia de eventos que se estudia para comprobar la hipótesis comienza por los orígenes de la política exterior emiratí desde la independencia en 1971 . Después, el trabajo se centra en el contexto circundante a las revueltas árabes de 2011 y a los factores regionales e internacionales que han motivado esta creciente independencia frente a su aliado Arabia Saudí. Finalmente, la secuencia temporal termina con la evolución de los escenarios regionales en los que EAU ha estado involucrado.

A nivel teórico, el trabajo se enmarca en la reformulación de la teoría de la dependencia de Hanieh (2019), que propone que el Golfo supone un nodo fundamental en una estructura reticular de dependencias en el sistema internacional, mostrando además la relación entre política exterior y economía política dentro 
del marco del Estado. Hanieh cuestiona, por tanto, la dicotomía centro/periferia, y plantea que la estructura de dependencias no es binaria (norte/sur, centro/periferia, primer/tercer mundo, etc.), sino reticular, tejida en torno a nodos con más o menos conexiones y roles distintos en el sistema, también con distintos componentes geográficos. En este sentido, según los modelos clásicos de dependencia (Amin, 1974; Wallerstein, 2004), los países centrales son Estados industrializados, mientras que la periferia son países que fundamentalmente exportan materias primas que los países centrales demandan. El sistema crea una serie de dependencias de la periferia hacia el centro. Esta aproximación ha recibido diversas críticas que apuntan a que el comportamiento de los actores a nivel internacional, aun dentro del marco de la dependencia, adquiere muy distintas formas (Hey, 1993). Otras formulaciones teóricas apuntan en una dirección similar, como el realismo subalterno de Ayoob (2002), que subraya la importancia de la expansión del neoliberalismo a la hora de definir la estructura de dependencias internacionales y que vincula el orden doméstico e internacional. En un tono similar, Hanieh (2019) establece que esta estructura de relaciones es multinivel, y que por ello el Estado sigue siendo un actor válido a la hora de analizar las dinámicas en las que participa.

Este artículo se inserta en este debate y sugiere que el Golfo constituye un nodo de particular importancia en el actual sistema internacional, en el que el modelo político que representa EAU busca armonizar sus intereses nacionales con las coordenadas actuales de la globalización en su zona de influencia. La acumulación de capital y el exceso de liquidez provoca que actores que en modelos clásicos de dependencia serían periféricos tengan incentivos para asumir una mayor iniciativa en aspectos políticos e incluso militares, y adquirir así un protagonismo creciente. Este rol remite de nuevo al modelo reticular si se tiene en cuenta que ha sido fomentado también por EE. UU. a través de su estrategia en los países en los que interviene EAU, como Siria o Yemen, y coordinando acciones diplomáticas, como con los acuerdos de normalización con Israel. Por todo ello, EAU constituye un actor fundamental en un nodo de creciente importancia, con cada vez más estrechas conexiones con el resto del mundo y con Oriente Próximo, y que además se encuentra en un momento en el que varios actores disputan su preeminencia.

Frente a aproximaciones binarias, el Golfo supone un ejemplo de nodo que coconstituye el tejido global de poder político y económico, influyendo en los demás actores y regiones a la vez que estos influyen en él, cada uno en función de sus sistemas económicos y políticos. Para el caso de EAU importan especialmente los vínculos con EE. UU. y cómo ha cambiado su rol como exportador de petróleo y su creciente influencia en Oriente Próximo. Este trabajo contribuye, por tanto, al modelo de la dependencia demostrando que las relaciones económicas y políticas de EAU con el resto del mundo árabe siguen un patrón de expansión al apoyar a bloques antiislamistas, ya sea a nivel militar cuando se encuentra un conflicto abierto como en Yemen, a nivel político como en Egipto, o a nivel económico como con Israel. 


\section{LA POLÍTICA EXTERIOR DE EAU TRAS LA INDEPENDENCIA}

\section{Los tres pilares: alineamiento con Arabia Saudi, dependencia militar de EE. UU. $y$ politica de contención en el Golfo}

Tras la independencia de Gran Bretańa en 1971, la alianza de los siete emiratos que componen los EAU adoptó una diplomacia de tono moderado basada en tres aspectos: mantener la estabilidad regional, fortalecer sus vínculos con el resto de países de la península arábiga y desarrollar una política común, en gran medida influida por Arabia Saudí (Salame, 1981: 336). Salvando excepciones puntuales relacionadas con el expediente palestino o la cuestión iraní, EAU ha actuado durante décadas en consonancia con Arabia Saudí en casos como Iraq (1991), Bahréin (2011) o en la aproximación a los talibanes en Afganistán y, posteriormente, la inclusión en la alianza internacional forjada por EE. UU. en 2001 para derrocarlos. Sin embargo, las controversias territoriales históricas de Arabia Saudí con EAU y otras monarquías locales, en especial el sultanato de Omán, han sido frecuentes, como sucedió hasta 1974 con los territorios alrededor del oasis de Buraimi (Pike, 1994: 197-198).

Por su parte, EE. UU. se comprometió desde 1971 a defender a los Estados de la península arábiga, desprovistos de ejércitos e industrias militares competentes, frente a las amenazas externas, ya emanasen del bloque comunista o de las reclamaciones históricas de Irán o Iraq. Por ello, en el contexto de la Guerra Fría la cuestión de la seguridad en el Golfo se convierte ante todo en un asunto militar enmarcado en una política basada en dos pivotes: Israel y el Irán anterior a la Revolución islámica de 1979. Así, Washington ha tendido a disociar la seguridad de las petromonarquías de la del resto de Oriente Próximo, en un compromiso dictado por los «intereses y no los valores» y sustanciado en la protección de los yacimientos de hidrocarburos y las rutas comerciales desde la región hacia el mar Rojo y el océano Índico, así como una actitud complaciente ante el deficiente desempeño de estas monarquías en materia de derechos humanos y avances democráticos (Sadeq Shurab, 1987: 170-171). La caída del régimen del Shah en 1979 desajustó este enfoque estadounidense, que a partir de entonces incidió en la militarización del Golfo. Aprovechando su relevancia geoestratégica con acceso a los golfos Pérsico y de Omán y al océano Índico, EE. UU. ha establecido bases militares tecnológicamente muy avanzadas como Jebel Ali o Al Dhafra. EAU es, además, el segundo mayor cliente de material militar estadounidense ${ }^{1}$. Algunos amagos por parte de Irán de cerrar el estrecho de Ormuz, por donde transita un $35 \%$ del comercio marítimo de crudo, han puesto de manifiesto la relevancia de esta estrategia ${ }^{2}$.

1. Luck, Taylor. 2019. «New Arab military force to reckon with as "Little Sparta" rises», The Christian Monitor, 28-2-2019. Disponible en: https:/cutt.ly/XmdbidM.

2. Iddon, Paul. 2018. "A history of Iranian threats to blockade Gulf oil exports», The New Arab, 13-12-2018. Disponible en: https://cutt.ly/QmdbgkG. 
La relación de EAU con Irán, sin embargo, es más compleja. Por un lado, algunas fricciones territoriales ya existían antes de 1979 y se sumaron a la crisis regional, como la disputa por la soberanía de las islas de Tunb al-Kubra, Tunb al-Sugra y Abu Musa (Hellyer, 2001: 167). Por otro, las diferencias respecto de la presencia estadounidense en la zona o sobre el papel del islam político no han impedido que emiratos como Dubai deban en buena medida su florecimiento mercantil y financiero a sus robustos vínculos con la economía iraní, y que el Gobierno central no haya renunciado nunca a un canal de intermediación con Teherán. Recientemente, con la crisis originada por la COVID-19, EUA envió ayuda sanitaria a Irán, en un gesto que no fue secundado por otros Estados del Golfo. Del mismo modo, EAU ha intentado mantenerse al margen de una hipotética escalada de tensión entre iraníes y estadounidenses alentada por la Administración Trump.

\section{EAU y el Consejo de Cooperación del Golfo}

Los EAU desempeñaron una función primordial en el diseño y gestación del Consejo de Cooperación del Golfo (CCG, rebautizado posteriormente como Consejo de Cooperación para los Estados del Golfo), constituido en 1981, precisamente en Abu Dhabi. El CCG, conformado por Arabia Saudí, EUA, Catar, Bahréin, Kuwait y Omán, tenía una vocación económica y social. El emir Zayed ben Sultán, primer presidente de EAU, abogó durante años por una mayor integración entre los países socios, en ámbitos como el nacional y el político. Sin embargo, el CCG no ha dado pasos sustanciales en tal dirección; tampoco ha acometido transformaciones radicales en el ámbito comercial, bancario o monetario, y permanece como un proyecto siempre en "continuo desarrollo", con diferencias a nivel arancelario o en los sectores bancario y de inversiones (Kazzi, 2017: 150-151). Sumado a las diferencias políticas, el CCG comenzó a dibujar dos bloques: uno representado por Arabia Saudí, EAU y Bahréin; otro por Qatar, cuyos posicionamientos sobre las revoluciones árabes y sus conexiones con los Hermanos Musulmanes (HH. MM.), Hamás y Turquía suscitaban polémicas continuas. El posterior embargo impuesto por esos tres países a Catar en 2017 demostró la falta de unanimidad en el seno del CCG: Omán y Kuwait se mantuvieron al margen y abogaron por una solución negociada «entre hermanos».

El CCG tampoco muestra unanimidad sobre el modo de obrar con Irán. Aunque su documento fundacional se refería a necesidades políticas y económicas, sin mencionar expresamente a la Revolución islámica, el temor a una posible expansión islamista chií estaba en su propio origen. Así, con el estallido de la guerra irano-iraquí en 1980, Teherán acusó a las monarquías del Golfo de azuzar a Bagdad contra Irán. Sin embargo, algunos Estados como Omán nunca dejaron de mantener relaciones amistosas con Irán, lo que se constató en su capacidad de mediación entre Irán y el G5+1 en torno al uso de su programa nuclear (2013). Catar, por su parte, desarrolló proyectos económicos conjuntos, incluida la explotación del campo de gas «septentrional», el mayor del mundo. Los mismos EAU, a pesar de la hostilidad oficial hacia 
la república islámica, se han beneficiado de florecientes proyectos de inversión: los intercambios comerciales entre el emirato de Dubai e Irán representaban en 2017 el $90 \%$ del volumen comercial iraní con el mundo árabe; en 2013, el 96,7\% de las exportaciones de la península a Irán procedían de EAU, con transacciones valoradas en 15000 millones de dólares, a pesar de las sanciones estadounidenses a Irán ${ }^{3}$.

\section{EUA ante los grandes conflictos regionales en el Golfo}

La región del Golfo ha sido testigo de tres grandes conflictos bélicos entre 1981 y 2003. En el primero, protagonizado por Iraq e Irán (1980-1988), EAU comenzó apoyando a Iraq por «medios políticos», aportando financiación y cobertura diplomática siguiendo las directrices estadounidenses (Sadeq Shurab, 1987), si bien su diplomacia defendió una postura neutral. La progresiva ambivalencia de Washington hacia los dos bandos en litigio deparó numerosas fluctuaciones y la sensación, compartida por iraquíes e iraníes, de que los estadounidenses perseguían una guerra prolongada de desgaste. Saddam Husein despertaba casi tantas reticencias como la nueva república islámica debido a sus escarceos con la Unión Soviética y las proclamas expansionistas, especialmente inquietantes para el gobierno kuwaití. La asistencia prestada por EAU y otras monarquías permitió que Iraq sostuviera el pulso al ejército iraní, mucho más voluminoso y mejor entrenado desde la época del Shah. El buen hacer diplomático emiratí contribuyó a favorecer la imagen de mal menor a favor de Saddam Husein ante numerosos Estados occidentales, y al finalizar la contienda ayudó a la reconstrucción del país prestando dinero. Al mismo tiempo, Irán pudo acceder a las armas y la liquidez negados por los embargos internacionales gracias a que los conductos financieros y comerciales siguieron abiertos con EAU, sobre todo a través de Dubai (Adib-Moghaddam, 2007).

En el segundo gran conflicto, generado por la invasión iraquí de Kuwait en 1990, EAU sustentó la opción saudí de una gran coalición internacional liderada por EE. UU., aportando contingentes aéreos. Bagdad los acusó de participar en la guerra sucia entablada por Kuwait contra Iraq a través de la bajada de los precios del crudo y la exigencia de que los iraquíes devolviesen las deudas contraídas durante el conflicto. La intervención armada expulsó a los iraquíes de Kuwait y dio paso a las sanciones económicas contra Iraq, que supusieron el declive de su poderío militar. Esto produjo a su vez la emergencia de Irán como primera potencia regional y la asunción por parte de EAU de desarrollar sus fuerzas armadas. El tercer gran conflicto se plasmó en la invasión de Iraq en 2003 por parte de EE. UU., que contó con menor respaldo árabe e internacional en comparación con la intervención de 1991. EAU, al igual que sus vecinos del Golfo, permaneció en un discreto segundo plano, si bien aportó generosa

3. Financial Tribune. 2017. «Upward Trend in Iran-UAE Trade Transactions», 23-5-2017. Disponible en: https://cutt.ly/wmdnefm. 
cobertura económica y apoyo diplomático a Washington en foros regionales como la Liga Árabe.

En suma, durante las primeras décadas posteriores a la independencia, la política exterior de EAU estuvo fundamentalmente centrada en cuestiones que afectaban de manera directa al propio país o al Golfo, y en caso de participar en dinámicas regionales siguió las líneas marcadas por EE.UU. y las conveniencias saudíes. En esta primera etapa, por tanto, EAU no supone un nodo intervencionista en el tejido de relaciones global o regional como sucederá tras las Primaveras Árabes de 2010-2011.

\section{ESPARTANISMO, ISLAM POLÍTICO Y DESPERTAR DE LA FUERZA MILITAR}

\section{La adecuación a la Administración Obama (2009-2017)}

El 4 de junio de 2009, el presidente Obama anunció en un discurso en El Cairo las directrices de la que iba a ser su política exterior, abogando por un menor intervencionismo militar en oposición a la política exterior de George W. Bush, caracterizada por la guerra contra el terrorismo y la intervención en Iraq como ejemplo de imperialismo en el siglo XxI. A pesar de reconocer el potencial transformativo de las Primaveras Árabes de 2010-2011, la Administración Obama no se pronunció sobre la brutal represión en las protestas en Bahréin, la intervención de Arabia Saudí en Yemen, el colapso de Libia o la involución democrática en Egipto. Ante esta falta de claridad, contrariado además porque se hubiera dejado caer a Mubarak, Mohammed bin Zayed (MbZ), príncipe heredero desde 2004, definió a Obama como un presidente en quien no se podía confiar (Lynch, 2015).

La notable actividad de Yousef al-Otaiba, embajador emiratí en Washington desde 2008, ha sido determinante en la adecuación de la política exterior de EAU a la de EE. UU., haciendo que el mensaje de $\mathrm{MbZ}$ contra el islam político de grupos como al-Islah en los propios EAU, los HH. MM. en Egipto, Hamás en Palestina y en general los grupos apoyados por Qatar, permease en los círculos políticos de EE. UU., no solo en el Partido Republicano (Krieg, 2019: 103). Para EAU, las acciones y las consecuencias de la política exterior estadounidense influyeron de manera determinante a la hora de configurar su propia agenda. La doctrina Obama en Egipto y Túnez supuso la caída de dos dictaduras y la victoria electoral de los islamistas; en Libia, la desestabilización del país, en la que también colaboraron las fuerzas emiratíes, abrió camino para la aparición de milicias yihadistas; en Siria, la incapacidad para responder a las líneas rojas que había establecido Obama favoreció asimismo al yihadismo y dejó espacio a Rusia para marcar la agenda. Por otro lado, al-Otaiba llama la atención sobre las aportaciones de EAU a las campañas militares estadounidenses en Afganistán, Somalia, Libia o Siria e Iraq (guerra contra el Estado Islámico) o a las misiones de paz (Balcanes o Líbano), en aplicación de una máxima de la diplomacia emiratí de hoy: que el «Oriente Próximo moderado actúe» (al-Otaiba, 2014). 


\section{La militarización y el proyecto de Esparta}

La progresiva retirada de EE. UU. de Oriente Próximo y las protestas en los países árabes a partir de 2011 motivaron un reforzamiento de la presencia de los países del Golfo en la zona. Arabia Saudí, EAU y Catar tenían el capital, el poder militar y la voluntad de intervenir para expandir su radio de acción. EAU optó por la militarización, lo cual llevó al general James Mattis, secretario de Estado de Defensa entre 2017 y 2019, a referirse a EAU como «la pequeña Esparta», por su apuesta por la presencia militar en países que ahora considera trascendentales. Los bases de Berbera o Asaab, por ejemplo, que reproducen las posiciones del imperio omaní en África del Este, ilustran esta política ${ }^{4}$.

Krieg (2019: 92) señala que esta progresiva militarización comenzó en los años noventa del pasado siglo como parte de un plan más amplio de modernización y desarrollo que tuvo su punto de inflexión con el ascenso al poder de $\mathrm{MbZ}$ tras el infarto de su hermano Jalifa en 2014, y compara los casos de Qatar y Abu Dhabi: mientras que la estrategia del primero se asocia al desarrollo de una política de soft power, el segundo opta por la securitización. Oficiales del ejército egipcio asesoraron a los líderes emiratíes en esta estrategia, que incluía una creciente presencia de la mujabarat o policía secreta (ibid:: 96). A partir de 2014, MbZ se convirtió en el dirigente de facto de EAU: concentró en sus manos las tres grandes carteras (Defensa, Exteriores e Interior) y consagró el definitivo ascenso de Abu Dhabi sobre Dubai, debilitado por la crisis financiera de 2008. Para afianzar su posición, creó una fuerza militar autónoma de 60000 efectivos y servicio militar obligatorio desde 2014, y promovió su propio plan de desarrollo integral, denominado Ghadan 21 y dotado con 5000 millones de dólares, en un contexto de incremento exponencial en la compra de material militar ${ }^{5}$. Algunos movimientos de $\mathrm{MbZ}$, sin embargo, han despertado suspicacias en Washington, como los intentos de evitar una implicación directa en un enfrentamiento militar entre EE. UU. e Irán o la incorporación a la guerra de precios en los hidrocarburos de los saudíes contra los rusos en la primavera de 2020, que resultó contraproducente para el sector petrolífero estadounidense.

\section{La influencia de las relaciones con Rusia y China}

La relación entre Rusia y EAU está definida por los intereses comunes en Oriente Medio y tiene claras motivaciones económicas. Así, la visita del presidente Vladimir Putin y de una delegación de oficiales del Gobierno y empresarios

4. The Economist. 2017. «The Gulf s Little Sparta» 6 de abril. Disponible en: https://cutt.ly/ dmdnFtr.

5. Luck, Taylor. 2019. «New Arab military force to reckon with as "Little Sparta" rises», The Christian Monitor, 28-2-2019. Disponible en: https://cutt.ly/XmdbidM. 
rusos a Abu Dhabi en 2019 escenificó un afianzamiento de relaciones que se ha venido produciendo especialmente a partir de 2015, cuando EAU apoyó la intervención rusa en Siria. A nivel comercial, la visita resultó en un acuerdo comercial de 1,3 billones de dólares centrado especialmente en los sectores de la energía, la tecnología y la salud ${ }^{6}$. Por otro lado, Rusia participa del lado de EAU en conflictos como Libia, a la vez que EAU apoya a Rusia en su disputa con Turquía en el norte de Siria.

El caso de China viene determinado por la Nueva Ruta de la Seda (o Belt and Road Initiative, en inglés), un proyecto de infraestructura que pretende desarrollar un conector comercial global a través de una ruta marítima y una terrestre. Los puertos de EAU son parte fundamental de este proyecto, y China, que se sirve de EAU como uno de los principales nodos comerciales para distribuir sus productos en Oriente Medio, ha estrechado relaciones con ellos para invertir en su desarrollo logístico. La colaboración en el marco de la Nueva Ruta de la Seda fue establecida en 2012, y en 2018 el presidente Xi Jingping visitó EAU para profundizar estos acuerdos. El desarrollo en Abu Dhabi del puerto y sector industrial Khalifa, uno de los conglomerados más desarrollados del mundo, por ejemplo, se enmarca en este proyecto (Fulton, 2020).

El marco de relaciones de EAU con Rusia y China se define en términos económicos y políticos. En ambos casos, la estructura reticular en las relaciones de EUA con Rusia y China, tanto de forma directa como en los escenarios en los que todas estas potencias intervienen, mientras se mantiene la relación con EE.UU., demuestra la reformulación de la teoría de la dependencia en la que se basa este artículo y refuerza la importancia de EAU como actor en el marco global, más allá del discurso centro/ periferia, y la validez del Estado como marco de análisis. Así, la creciente relación de EAU con China y Rusia da cuenta de la creciente multipolaridad en Oriente Medio, el menor intervencionismo de EE. UU., el ascenso de China y las ambiciones de EAU y las monarquías del Golfo.

\section{La colisión con el islam politico}

Uno de los pilares de la agenda emiratí reside en la oposición frontal al islam político. MbZ mantiene una enemistad particular con los movimientos islamistas, motivadas cuando menos por la necesidad de garantizar la estabilidad interna. Como señala Freer (2018), la presencia del islamismo en el Golfo, y de los HH.MM. en concreto, no está institucionalizada como sucede en otros países árabes como Egipto o Túnez. Sin embargo, el reciente encarcelamiento de varias decenas de seguidores del Islah, la

6. Aljazeera. 2019. «Russia's Putin signs deals worth \$1.3bn during UAE visit», 15-9-2019. Disponible en: https://cutt.ly/vmdmo2i. 
perseguida franquicia local de los HH. MM., demuestra la preocupación del Gobierno por el islamismo ${ }^{7}$.

En consecuencia, la intervención de EAU en países como Egipto, tras la victoria en 2012 del candidato de los HH. MM., Muhammad Morsi, debe analizarse con respecto a esta dimensión interior. Los HH. MM. son una organización con presencia en la mayor parte de los países árabes, y con apoyos en países de mayoría musulmana como Turquía. Por lo tanto, evitar que esta red de franquicias se extienda a un país tan significativo a nivel político, económico y cultural como Egipto no era solamente una prioridad en el ámbito exterior para EAU, sino que tenía un efecto en su estabilidad interna. Tal como señaló Anwar Gargash, ministro de Exteriores emiratí entre 2008 y 2021, la oposición al islamismo ha sido una de las líneas maestras de la política exterior emiratí, especialmente tras las Primaveras Árabes ${ }^{8}$.

\section{La rivalidad interna entre Abu Dhabi y Dubai}

El incontestado protagonismo de MbZ en la federación no puede explicarse sin las vicisitudes del binomio Dubai-Abu Dhabi, gobernados por los Al Nahyan y los Al Maktum, respectivamente. El caso de Dubai constituye a la vez una historia de éxito y fracaso. Siendo originalmente un emirato a la sombra de Abu Dhabi (Davidson, 2008: 103), con muchos menos recursos fósiles, la estrategia de diversificación consiguió que Dubai se convirtiera eventualmente en la cara visible de EUA, con la figura de Mohammed bin Rashid Al Maktum como principal valedor. El proyecto de Dubai consistió en convertirse en una metrópolis cosmopolita con uno de los puertos con más tráfico del mundo, una industria turística de lujo y un mercado inmobiliario y financiero boyante, así como un modelo urbano basado en el mall o centro comercial y que ha sido exportado a otros países bajo la etiqueta de «dubaización» (Elshestawy: 2009). Sin embargo, tal y como Davidson (2008) predijo, el modelo de Dubai ha ido cayendo en desgracia, particularmente tras la crisis de 2008, que motivó la inyección de diez billones de dólares desde Abu Dhabi. Además, el rescate consolidó el control político por parte del emirato vecino?.

La pérdida de poder de Dubai frente a Abu Dhabi, especialmente a partir de 20082009, da cuenta de cómo la estrategia exterior de EAU ha ido adaptándose a los designios de $\mathrm{MbZ}$ y no tanto a los de Al Maktum o los otros cinco emiratos. Más aún, la

7. Aljazeera. 2013. «UAE coup plot trial begins in Abu Dhabi», 4-3-2013. Disponible en: https:// cutt.ly/mmdQAoL.

8. Gargash, Anwar. 2012. «Amid challenges, UAE policies engage gradual reforms», The National, 26-8-2012. Disponible en: https://cutt.ly/HmdQFKn.

9. Thomas, Landon Jr. 2009. «Abu Dhabi Tightens its Grip as It Offers Help to Dubai», The New York Times, 14-10-2009. Disponible en: https://cutt.ly/pmdE5Mj. 
pérdida de relevancia de Dubai ha acentuado la centralización del país y, por tanto, de su política exterior.

\section{Soft power, Estado de la vigilancia y estrategia de presión múltiple}

La gestión del capital que produce una economía fundamentalmente basada en petrodólares constituye una forma de política exterior que en el caso de EAU adquiere dos vertientes: por un lado, la relación entre la economía política emiratí y la global (Hanieh 2019:7), y entre EAU y Oriente Próximo, por otro. El auge económico de EAU, especialmente a partir de los 2000, ha permeado y transformado las redes del capitalismo global y constituye un nodo en sí mismo de relaciones con el resto de economías del globo, sea como proveedor, inversor, donante, business partner o potencia neocolonial. En relación con este papel financiero y amparándose en sus diversos conglomerados industriales, EAU ha venido exportando la idea de seguridad e ingeniería social que MbZ ha querido desarrollar para Abu Dhabi (Jones, 2017: 37). En el marco de unas relaciones difusas entre Estado y capital privado, el capitalismo de la vigilancia que describe Zuboff (2019) se extiende a través de proyectos de smart cities en la región, con proyectos en marcha en Egipto, Jordania, los territorios palestinos o el Cuerno de África, promoviendo a su vez una noción de seguridad asociada a la agenda exterior de EAU, como el apartado anterior señala. Tienen un papel preponderante las empresas de telefonía emiratíes, que proveen la infraestructura y servicios de este modelo urbano basado en la extracción de datos (Hanieh, 2019: 87-89). A su vez, este nuevo modelo de Estado de la vigilancia supone una variable importante a la hora de entender la creciente relación entre EAU e Israel, basada en el intercambio de tecnología y servicios dentro del sector ${ }^{10}$.

Otra de las estrategias exteriores de EAU ha sido lo que Nye (2012) denominara soft power, definido como la capacidad de influir en otro agente sin emplear coerción ni influencia económica. De nuevo en relación con las rentas del petróleo, la inversión en museos, festivales y arte en general representa la pretensión de convertir el capital económico en capital cultural, y supone una forma de posicionar al país en la vanguardia del mundo de la cultura. La proliferación de bienales, megaeventos o museos pretende ocupar el vacío que dejó el fracaso del panarabismo. Algunos ejemplos de estas políticas culturales incluyen la apertura de museos como el Louvre o el Guggenheim de Abu Dhabi, el partenariado entre dicho emirato y la New York University, las ferias Art Dubai o la Bienal de Sharjah, la celebración de la Copa Asiática 2019 o la Expo Dubai 2020, cancelada por la pandemia de la COVID-19. A nivel político, la estrategia ha consistido en la creación de un Ministerio de la Felicidad, un Ministerio de la Tolerancia y un Ministerio de la Juventud, este último a cargo de una

10. Donaghy, Rori. 2015. «Falcon Eye: The Israeli-installed mass civil surveillance system of Abu Dhabi», Middle East Eye, 15-7-2015. Disponible en: https://cutt.ly/1mdRQWu. 
mujer de veintidós años ${ }^{11}$, como forma de distanciarse de la imagen de petro-Estado autoritario que prácticas como las condiciones laborales de los trabajadores extranjeros puedan proyectar.

\section{LA NUEVA POLÍTICA EXTERIOR DE UAE TRAS LA PRIMAVERA ÁRABE}

A medida que las protestas iban ganando fuerza en varios países árabes, en EAU se agravó el temor al ascenso del islam político sunní (HH. MM.) o chií (activistas motejados de proiraníes en Bahréin, Hezbolá en Líbano y Siria, las milicias progubernamentales en Iraq y los Huzíes en Yemen). Para Abu Dhabi, la caída de dirigentes autoritarios durante la primera mitad de 2011, en especial Ben Ali y Mubarak, supone un motivo de preocupación ante el impulso de formaciones como Ennahda tunecino o los HH. MM. egipcios y un acicate para extender la doctrina MbZ en Oriente Medio. A partir de aquí, los intereses exteriores y de economía política van a motivar no solamente una expansión del rol de EAU en Oriente Medio, sino la adquisición de un papel que determina el mapa de relaciones con y entre los demás actores de la región, por una parte, y el resto de potencias globales, por otro.

La hostilidad entre las principales monarquías del Golfo y la Hermandad remite a las tensiones interárabes generadas por la invasión de Kuwait y posterior campaña bélica contra Saddam Husein, y a la batalla doctrinal e ideológica entre el wahabismo, que sustenta la legitimidad religiosa intraislámica de la familia saudí, y un islamismo político mucho más activo en el ámbito nacional y regional. Los HH.MM., además, defienden el acercamiento a Turquía, la negociación con Irán y la reivindicación inequívoca de la cuestión palestina, tres asuntos especialmente problemáticos para emiratíes y saudíes.

En el ámbito interno, el régimen contuvo sin miramientos la tímida contestación producida al calor de la Primavera Árabe, organizada en torno a una rama liberal, de tendencia secularista y occidentalizante, y otra islamista, asociada al reformismo islamista señalado antes (Davidson, 2013: 220-226). En este sentido, y en contraste con otros países árabes, el robusto liderazgo político emiratí sí disponía de instrumentos financieros y liquidez monetaria suficientes para aplacar las posibles demandas de sus súbditos y asegurar una suerte de paz social a base de subsidios, exenciones de impuestos y empleos en el sector público.

\section{La contención estratégica en el Golfo: la intervención en Bahréin}

El concurso de EAU en la intervención armada saudí en Bahréin en marzo de 2011, en el marco de la operación Escudo de la Península Arábiga, supone la primera

11. Espinosa, Ángeles. 2016. "Posiblemente, la ministra más joven del mundo», El País, 11-22016. Disponible en: https://cutt.ly/xmdRYKG. 
acción de envergadura dentro de su nuevo planteamiento estratégico regional. De manera similar a lo sucedido en Egipto y Túnez en meses anteriores, Bahréin fue testigo de una serie de movilizaciones y concentraciones populares, concentradas en la plaza de la Perla de Manama. Sus demandas incluían más poderes para el Parlamento, libertad de expresión, liberación de presos políticos y delimitación de las competencias del rey y sus familiares. Las redes sociales, como en otras revoluciones árabes, fueron fundamentales para convocar a los manifestantes y facilitar su comunicación (Bassiouni et al., 2011: 65-66). A las protestas se sumaron sunníes, chiíes, islamistas de ambas ramas e izquierdistas, aunque como señala Matthiesen (2013: 12), la mayor parte de los manifestantes eran chíes; del mismo modo que la mayor parte de los habitantes de Bahréin también lo son. La represión, torturas incluidas, ejercida por las autoridades y las fuerzas del Consejo de Cooperación del Golfo, tuvo un componente sectario dirigido hacia la comunidad chií (Bassiouni et al., 2011: 419).

En opinión de emiratíes y saudíes, y también de las potencias occidentales, el caso bahreiní difería del de otros países árabes. Para EAU, Bahréin está demasiado cerca como para permitir la caída de un Gobierno aliado, y el cuestionamiento del liderazgo en Manama podía tener efecto dominó en la zona, peligroso para la legitimidad de las autoridades emiratíes. El CCG envió efectivos en marzo, dentro de la citada operación, con el cometido oficial de proteger instalaciones sensibles, como los pozos de petróleo, si bien las tropas participaron en la represión de las protestas. Arabia Saudí y EAU proporcionaron el grueso del contingente — quinientos policías por parte emiratí-, al que se sumarían efectivos navales kuwaitíes, y potenciaron una batalla mediática en la cual las protestas se describían como producto de maniobras iraníes en favor de los chiíes, incidiendo así en la narrativa sectaria ${ }^{12}$.

La intervención tuvo éxito: Bahréin estableció la ley marcial e impuso medidas represivas contra activistas y manifestantes. Desde entonces, Arabia Saudí ha ejercido un control político y militar férreo con el objetivo de garantizar la estabilidad política y preservar los intereses económicos. Esta política cuenta con el apoyo de EAU (Barany, 2016) y supone el pilar de una estrategia de contención frente a la proyección iraní sobre el Golfo.

\section{Siria y el progresivo acercamiento a al-Asad}

En Siria, aunque la posición de EAU había sido la de evitar el crecimiento del islamismo político, la enorme dispersión de fuerzas en el conflicto y los innumerables intereses cruzados han hecho esta tarea imposible. Ejemplo de ello es la estructura del Ejército Libre Sirio, formado en 2011, que a partir de 2012 entra en un estado de

12. Noueihed, Lin y Frederik Richter. 2011. "Saudi sends troops, Bahrain Shi' ites call it 'war'», Reuters, 14-3-2011. Disponible en: https://cutt.ly/VmdTiQp. 
fragmentación, con milicias seculares e islamistas enfrentadas entre sí y apoyadas por valedores externos contrapuestos entre sí.

Asimismo, el conflicto ha pasado por varias fases que han hecho que los apoyos de EAU cambiasen en función del desarrollo de los acontecimientos, aunque siempre ha mantenido su rivalidad hacia los HH. MM. y grupos afines. Al contrario que Arabia Saudí y Qatar, que sustentaron de forma más sostenida a las milicias anti-Asad, incluidos grupos islamistas y salafistas como Ahrar al-Sham o Yaysh al-Islam ${ }^{13}$, EAU, a pesar de un discurso oficial beligerante, mantuvo una relativa cordialidad con el régimen de Asad. El progresivo acercamiento de EUA a Damasco responde a la evidencia de que esta, protegida por Rusia e Irán y tras dejar EE. UU. bien claro que su prioridad era combatir al yihadismo internacional y no a al-Asad, se encuentra en una posición ventajosa. Por ello, se trataba de contrarrestar la influencia de Irán intentando mantener un contacto directo con al-Asad ${ }^{14}$, y de maniobrar respecto a Turquía, tal como muestran las alianzas con los peshmerga y Moscú en el Kurdistán sirio ${ }^{15}$.

\section{Libia y el salto cualitativo de la nueva politica intervencionista}

En el expediente libio, el año 2014 marca un importante cambio por el creciente apoyo al mariscal Haftar, enfrentado a las milicias islamistas y al Estado Islámico en el este (Cirenaica) y al Gobierno de al-Sarraj en el oeste (Tripolitania). Los eventos que tuvieron lugar en 2011 en Libia constituyeron una revolución política en toda regla, dado que además de retirar del poder a Gadafi, asesinado en octubre de ese año, se construyó un nuevo Estado. Durante los primeros meses de alzamiento, la posición de EAU se atuvo a la de la Liga Árabe, que retiró la membresía de Libia en protesta por la represión ejercida por el régimen de Gadafi. Luego, apoyó la intervención de la OTAN en marzo de 2011 y la formación posterior de un Consejo Nacional de Transición.

La posición de EAU comenzó a variar tras las elecciones de 2012, en las que los partidos islamistas, de corte similar a los HH.MM., recibieron el apoyo mayoritario de los votantes ${ }^{16}$. El país entró en una dinámica de tensión permanente entre Trípoli y Bengasi y la expansión del yihadismo internacional. Abu Dhabi refrendó la operación al-Karama (Dignidad) lanzada por Haftar para limpiar Libia de HH. MM. y

13. Oweis, Khaled Y. 2013. «Insight: Saudi Arabia boosts Salafist rivals to al Qaeda in Syria», Reuters, 1-10-2013. Disponible en: https://cutt.ly/4mdTcoa.

14. Reuters. 2018. «UAE reopens Syria embassy in boost for Assad», 27-12-2018. Disponible en: https://cutt.ly/7mdTWfT.

15. Mardasov, Anton. 2020. «Russia explores way to draw UAE, Saudi Arabia to its Syria policies, explores way to draw UAE, Saudi Arabia to its Syria policies», Al Monitor, 27-2-2020. Disponible en: https://cutt.ly/kmdTTfF.

16. Stephen, Christopher. 2012. «Mahmoud Jibril seeks coalition with Libya's Islamists after his poll win", The Guardian, 14-7-2012. Disponible en: https://cutt.ly/tmdTOZ4. 
terroristas yihadistas. La infraestructura necesaria para llevar a cabo dicha operación, así como la mayor parte del armamento del ejército de Haftar, fue proporcionado por Egipto y EAU. La campaña de Haftar, lejos de pacificar el país, exacerbó las dinámicas de fragmentación y la lógica del intervencionismo externo, con numerosos actores internacionales implicados. Libia llegó a contar con tres Gobiernos al mismo tiempo, además de un sinfín de milicias armadas que recibían financiación y armas de una lista nutrida de potencias regionales e internacionales. Para EAU, la apuesta en favor de Haftar se insertaba en su campaña panárabe contra el islamismo político y el yihadismo. El caso libio supone un salto cualitativo, ya que constituye el primer ejemplo de intervencionismo militar de EAU fuera del CCG.

La diplomacia emiratí ha contribuido a forjar la imagen de un Haftar convertido en paradigma de un moderno secularismo árabe compatible con un islam apolítico y moderado, radicalmente hostil al yihadismo internacional y el islamismo político. Esta percepción ha favorecido que países como Francia, que tanto se implicó en la campaña militar contra Gadafi, mantengan una doble vía de colaboración con el Gobierno de unidad nacional apoyado por la ONU, pero también con Haftar (Badi, 2020). Sin embargo, los reveses sufridos por Haftar tras la ofensiva lanzada contra Trípoli en 2019 cuestiona la rentabilidad de la estrategia militar exterior de MbZ. Catar, y sobre todo Turquía, que apoyan decididamente al presidente al-Sarraj, habían conseguido nivelar la balanza frente al empuje inicial del trinomio emiratí-saudí-egipcio.

\section{Participación en la ofensiva saudi en Yemen: la visión propia sobre la estabilidad del Golfo}

El conflicto en Yemen es uno de los ejemplos más claros del espartanismo de EAU y su autonomía respecto de Arabia Saudí. La llamada Coalición Árabe que intervino militarmente en Yemen está liderada por Riad; sin embargo, EAU tuvo un papel relevante en la gestación de la operación Tormenta Decisiva. Según algunas fuentes, fue $\mathrm{MbZ}$ quien convenció a los saudíes de la necesidad de poner coto a la influencia iraní en el sur de la península (Brehony, 2020: 136). En marzo de 2015, tras las reiteradas peticiones del presidente yemení, Abdrabboh Mansur Hadi, y la preocupación en el Golfo ante los huzíes, que habían tomado Saná y marchaban hacia Adén, Arabia Saudí organizó una campaña de bombardeos para neutralizar lo que consideraba influencia iraní en su patio trasero. Con la aportación de Marruecos, Egipto, Bahréin y Catar, más el apoyo tácito o explícito de EE. UU. y otros países de la Unión Europea, la coalición restauró a Hadi en el poder.

Sin embargo, el conflicto evolucionó hacia un caos en el que un inmenso número de intereses y grupos se encontraban en disputa. En un contexto de tribus armadas con reivindicaciones propias, facciones del ejército leales al expresidente Saleh, aliado circunstancial de los huzíes, milicias secesionistas en el sur, al-Qaeda en la península arábiga (AQAP) y el Estado Islámico (ISIS), islamistas de al-Islah y 
militares partidarios del general Mohsen al-Ahmar, EAU ha ido focalizando su presencia en el sur, especialmente en el puerto de Adén, debido a su importancia comercial. En un primer momento, sus tropas entraron con ímpetu en el conflicto, asesoradas por veteranos militares occidentales y reforzadas por mercenarios de Colombia y otros países latinoamericanos ${ }^{17}$. En los últimos años han establecido una entidad paralela en la antigua República Democrática de Yemen, que se ha dedicado a combatir a los huzíes, a las tropas leales a Hadi y, especialmente, a las corrientes del islamismo político como al-Islah, desviándose así de la línea exclusivamente antiiraní marcada por Arabia Saudí ${ }^{18}$.

Otro ejemplo del unilateralismo emiratí frente a los saudíes fue el envío en 2018 de tropas al estratégico archipiélago de Socotra para hacerse temporalmente con el control de los accesos marítimos y aéreos. El Gobierno de Yemen, en respuesta pidió a Arabia Saudí que interviniese como mediador y, a la postre, tanto las tropas saudíes como las emiratíes permanecieron en el terreno, compitiendo por el control de las diferentes zonas y grupos sociales. En abril de 2020, de nuevo, el Consejo de Transición del Sur (CTS), que había expulsado temporalmente en agosto de 2019 a las facciones armadas leales a Hadi de Adén, declaró el autogobierno en el sur de Yemen, algo que la coalición liderada por Riad rechazó ${ }^{19}$. Días después, se produjeron altercados en Socotra entre contingentes armados proemiratíes y milicias afines a Arabia Saudí. La estrategia de Socotra perseguía asegurar el dominio estratégico del archipiélago para asentar el despliegue de EAU en el Cuerno de África.

La actuación de EAU, en apariencia lesiva para los movimientos saudíes, tiene que ver con el hecho de que estos han permitido la influencia de los islamistas de al-Islah -que a pesar de sus vínculos históricos con los HH. MM. ha mantenido una actitud amistosa hacia Arabia Saudí- en el gobierno de Hadi; pero, también, con la percepción de sus dirigentes de que Arabia Saudí no tiene capacidad para imponer una solución estable en Yemen (The Soufan Center, 2020). De hecho, el objetivo primordial de la coalición, derrotar a los Huzíes, dista de haberse alcanzado, y los emiratíes han dejado de enfrentarse directamente a estos sobre el terreno, en especial tras la muerte de 45 de sus soldados en Mareb, en 2015, que tuvo un gran impacto en su opinión pública. A partir de 2019, EAU redujo sus tropas y en noviembre de ese año un representante de Exteriores señaló que «los huzíes tendrán su papel en el futuro de Yemen» (Fadl, 2019), postura muy diferente de la mantenida al inicio de la campaña bélica. Todo ello desmiente el supuesto seguidismo emiratí a las consignas saudíes y confirma que Abu Dhabi tiene su propia hoja de ruta.

17. Mazzetti, Mark y Emily Hager. 2015. «Emirates secretly sends Colombian mercenaries to Yemen fight», New York Times, 25-11-2015. Disponible en: https://cutt.ly/ZmdYm9I.

18. Abdul-Ahad, Ghaith. 2018. «Yemen on the brink: how the UAE is profiting from the chaos of civil war", The Guardian, 21-12-2018. Disponible en: https://cutt.ly/xmdYR2U.

19. Aljazeera. 2020. «Saudi-led coalition rejects south Yemen self-rule declaration», 27-4-2020. Disponible en: https://cutt.ly/8mdYXYB. 
La apuesta por la baza regional de al-Sisi: Egipto, cabeza de puente de la politica árabe de EAU en el Mediterráneo

Egipto constituye una de las piezas centrales de la política exterior de EAU. En 2013, el general Abdel Fatah al-Sisi dio un golpe de Estado con el apoyo de Arabia Saudí, Kuwait y EUA, algo más de un año después de la victoria en las presidenciales de Mohammed Morsi, del partido Liberad y Justicia, brazo político de los HH. MM. Los tres países del Golfo apuntalaron a al-Sisi con una gran cobertura mediática y un respaldo de 20000 millones de dólares ${ }^{20}$. EAU ha favorecido numerosos acuerdos bilaterales a nivel económico y militar ${ }^{21}$.

Al-Sisi ha mantenido una política plenamente alineada con los intereses de EUA, como en el caso de Libia. Habida cuenta de la relevancia histórica de Egipto en el mundo árabe e islámico, el hecho de que saudíes y emiratíes mantengan una influencia directa sobre la toma de decisiones en el país del Nilo certifica el ascendente regional del Golfo. Egipto, además, supone un aliado fundamental para un proyecto de expansión en el Mediterráneo, sobre todo tras las ventajas obtenidas por la aviación emiratí para utilizar las bases militares egipcias en sus ataques aéreos contra los islamistas libios. A la par, la confluencia con El Cairo permite extender las relaciones cada vez más amistosas con Israel, sirviéndose de al-Sisi como intermediario para la normalización de relaciones entre Israel y muchos países árabes ${ }^{22}$.

Otro motivo en pro de la entente al-Sisi/MbZ tiene que ver con Turquía, la otra gran potencia regional en el Mediterráneo. Erdogan fue uno de los grandes valedores de Morsi, junto con Catar, y condenó su deposición y posterior encarcelamiento. Ankara se ha mostrado reacia al desembarco mediterráneo de EAU y su progresivo acercamiento a Israel. Por tanto, EAU tiene gran interés en mantener en Egipto un dique de contención frente a Turquía. En el mercado del gas, por ejemplo, Egipto ha abogado por una mayor integración con países como Chipre, Israel o Italia, dejando a Turquía al margen ${ }^{23}$. Sin embargo, al-Sisi afronta un creciente descontento popular que trata de neutralizar con medidas de coerción, control y censura, mientras que la propuesta estrella de su mandato, convertir Egipto en una potencia económica - la sexta del mundo para 2030 según la prensa oficialista local ${ }^{24}$ se ve lastrada por la lentitud de los proyectos de desarrollo, las tensiones con Etiopía en torno a la

20. Aljazeera. 2014. «Saudi King Abdullah visits Egypt’s Sisi», 20-6-2014o. Disponible en: https://cutt. $\mathrm{ly} / \mathrm{UmdUm} 4 \mathrm{~V}$.

21. Fanack. 2020. «As-siyasat al-jarijiyya li-l-Imarat al'Arabiat al-Muttahida, Qatar al-yadida?», 27-4-2020. Disponible en: https://cutt.ly/amdUOaS.

22. Black, Ian. 2019. "Why Israel is quietly cosying up to Gulf monarchies», The Guardian, 19-32019. Disponible en: https://cutt.ly/3mdU6V6.

23. Reuters. 2019. "Eastern Mediterranean countries to form regional gas market», 14-1-2019. Disponible en: https://cutt.ly/hmdItfl.

24. Al-Naba'. 2020. «Muasshirat tahassun wad' al-iqtisad al-misriyyi bidayat 2020», 19-2-2020. Disponible en: https://www.elnabaa.net/809797. 
explotación del Nilo, vital para Egipto, y los problemas de liquidez, apartados en los que la generosa ayuda financiera de EAU no puede ofrecer soluciones mágicas.

\section{La controversia con Catar y el difícil equilibrio en el Golfo}

En mayo de 2017 se dieron a conocer unos supuestos comentarios del emir Tamim bin Hamad Al Thani en la Agencia de Noticias de Catar en los que ensalzaba la relación del país con Irán, se mostraba comprensivo con Hezbolá y Hamás y sugería que Trump no duraría mucho como presidente de EE. UU. Doha las desmintió y achacó a una infiltración de piratas informáticos, que habían manipulado las palabras originales del líder qatarí. EAU, Arabia Saudí y el propio Trump las tomaron como ciertas, si bien fuentes de inteligencia estadounidenses apuntaron con posterioridad que el jaqueo se había producido desde EAU, extremo que el embajador al-Otayba negón ${ }^{25}$. Dos semanas después, ambas monarquías y Bahréin cortaron relaciones diplomáticas con Catar con el argumento de que Doha financiaba el terrorismo y mantenía relaciones sospechosas con Irán (Krieg, 2019: 3-4). Pronto se unirían otros Estados como Egipto o, en menor medida, Jordania.

No obstante, los conflictos entre saudíes y emiratíes por un lado, y cataríes por otro, no son nuevos. El ascenso al poder en Catar del emir Hamad Bin Jalifa Al Thani, en 1995, que planteó una política interior y exterior propia, alejada de las preferencias de Arabia Saudí, tensó las relaciones bilaterales. Incluso se acusó a Riad de orquestar un golpe de Estado contra Hamad en 1996 con la ayuda de la tribu Al Murra, que reside a ambos lados de la frontera ${ }^{26}$. EAU, por su parte, tenía otros motivos, vinculados como se ha apuntado ya con las revoluciones árabes y los nexos cataríes con el islamismo político. Para Abu Dhabi, Doha se había convertido en un rival cada vez más influyente en una zona geográfica de creciente interés (Roberts, 2014: 85). El bloqueo, por tanto, más allá de la supuesta connivencia de Catar con Irán o los alegatos de la televisión $\mathrm{Al} \mathrm{Jazeera}$ en contra de los dirigentes autoritarios árabes cercanos a Riad y Abu Dhabi, perseguía aislar a la diplomacia qatarí, exponer ante el patrón estadounidense su presunto doble juego y neutralizar la influencia turca en la región árabe. Así, este posicionamiento no tiene tanto que ver con las prioridades saudíes como con los vectores de la política exterior emiratí. Esta ha intentado contrarrestar la narrativa promovida por Catar de islamismo y democratización con un mensaje de islam moderado y tolerancia religiosa.

La eficacia del bloqueo ha sido dudosa, ya que ha empujado a Catar a estrechar sus vínculos comerciales y políticos con Irán y Turquía, superando la absoluta dependencia

25. Tharoor, Ishaan. 2017. "The Blockade of Qatar is failing», Washington Post, 18-7-2017. Disponible en: https://cutt.ly/zmdInVg.

26. Al Hakeem, Mariam. 2005. "Thousands in Saudi Arabia after losing Qatari citizenship», Gulf News, 3-4-2005. Disponible en: https://cutt.ly/xmdIPmx. 
de abastecimiento que la sujetaba a Arabia Saudí (Krieg, 2019: 3). Además, ha incomodado a miembros del CCG como Kuwait y Omán, no ha generado la animadversión de las potencias occidentales hacia Catar y no ha afectado a China y Rusia, que han estrechado sus relaciones con todos los estados del Golfo. En definitiva, Catar resistió el embargo sin alterar su política regional e internacional, lo cual ha perjudicado la imagen de fuerza que saudíes y emiratíes deseaban proyectar. En clave interna, el apoyo decidido de Abu Dhabi al bloqueo puso de relieve la pérdida de influencia económica y sobre todo política de Dubai, muy perjudicado por las sanciones internacionales a Irán y la abrupta interrupción de transacciones comerciales con Catar.

\section{La expansión en el Cuerno de África}

Los referidos movimientos en el archipiélago de Socotra forman parte del plan de EAU de extender su influencia al Cuerno de Âfrica. La posición estratégica de aquel, cercana también al mar Rojo, así como la inestabilidad generada por el conflicto yemení, la convirtieron en cabeza de puente del renovado interés emiratí en las costas africanas orientales. La dinámica expansiva de EUA en el Cuerno de África cambió de dirección tras la disputa diplomática con Omar Guelleh, presidente de Yibuti, en 2014. El CCG, sobre todo EAU, tenía permiso para administrar una base militar en el país - Haramous, al sur de la capital—, que se había convertido en objeto de deseo para actores como China o Turquía, esta última especialmente preocupante para $\mathrm{Abu}$ Dhabi por su consabida rivalidad con Erdogan. No obstante, el acercamiento de saudíes y emiratíes a Eritrea, enemigo de Yibuti, suscitó el enojo de Guelleh y la oposición de su Gobierno a facilitar los proyectos empresariales de compañías de EUA en puertos estratégicos como el de Dorale (African Intelligence, 2015). Abu Dhabi buscó entonces desplazar sus destacamentos militares, de vital importancia para el control del mar Rojo y el golfo de Adén, a Masawa y Asab, en la costa eritrea, bajo el paraguas del CCG (Mello y Knights, 2016). Esto permitió ampliar el marco de operaciones en Yemen y, de paso, enviar una advertencia a Etiopía, enemigo tradicional de Eritrea y enfrentado al Egipto de al-Sisi por los recursos hídricos del Nilo ${ }^{27}$.

Las negociaciones para ampliar las instalaciones militares y comerciales de EAU se extendieron también a Sudán (Port Sudan) y a Somalilandia (puerto estratégico de Berbera), tras los roces diplomáticos con Somalia, que se había negado a secundar el embargo a Qatar. En reacción a las presiones saudíes y emiratíes, Mogadiscio permitió en 2017 la apertura de una base militar turca para entrenar a su ejército, mientras que Doha ha impulsado proyectos de desarrollo en el país. Al tiempo, Ankara ha reforzado sus vínculos comerciales con Etiopía y, a través de Catar, trató de mejorar sus relaciones con el Gobierno de Omar al-Bashir en Sudán. La disputa entre Riad-Abu Dhabi-El

27. Fanack. 2020. «As-siyasat al-jarijiyya li-l-Imarat al'Arabiat al-Muttahida, Qatar al-yadida?», 27-4-2020. Disponible en: https://cutt.ly/lmfejL9. 
Cairo, por un lado, y Doha-Ankara, por otro, se redobló después de la revuelta popular que depuso a al-Bashir y propició el ascenso de una junta militar. Esta, por el momento, se ha decantado por el primer eje.

Para complicar la confusa relación de alianzas en la costa oriental del continente, China se ha apuntado a la competición por asentar instalaciones militares, lo que ha provocado la suspicacia de estadounidenses y europeos. Los movimientos de EAU vienen condicionados por el desarrollo de los conflictos yemení y libio y las maniobras de cataríes y turcos. Todo esto ha conformado un panorama de enorme complejidad en el que las potencias extranjeras tratan de obtener el favor de los Estados africanos, pobres y sumidos en crisis cuasi crónicas. EAU ha intentado expandirse incluso hacia la costa sudoccidental, a Namibia, a través de proyectos portuarios emprendidos por empresas de Dubai (Africa Fundación Sur, 2020).

\section{La nueva perspectiva sobre la cuestión palestina y la normalización de relaciones con Israel}

Uno de los cambios más notorios de esta política exterior se relaciona con la cuestión palestina. Desde hace años, han aumentado los contactos comerciales, diplomáticos e incluso militares entre Israel y EAU, lo que ha llevado a la ruptura del boicot tradicional de EUA y otros Estados árabes hacia Israel, la potencia ocupante de Palestina. En 2015 se abrió la primera oficina oficial del Estado de Israel en EAU ${ }^{28}$ en un marco de intercambios crecientes, centrados especialmente en los sectores de comunicaciones e inteligencia ${ }^{29}$. En un encuentro informal en la Casa Blanca, el embajador al-Otayba se reunió con un alto cargo de la Seguridad Nacional de Israel para abordar un pacto de no agresión y normalizar las relaciones en un futuro próximo ${ }^{30}$. El argumento central de la reunión era Irán, asunto que junto con Hamás, Hezbolá y el ISIS constituye un motivo de preocupación compartido. También está el poso de insatisfacción por las dinámicas de acercamiento de la Administración Obama a Irán entre 2012 y 2016, a la que emiratíes, saudíes e israelíes acusaban de no haber tenido en cuenta sus intereses en las negociaciones para el acuerdo nuclear ${ }^{31}$.

Por otro lado, la búsqueda de una entente con Tel Aviv responde a la retirada progresiva de EE. UU. de la región del Golfo, lo que reafirma su posición de nodo a nivel económico y político en la estructura global de dependencias. Por esta razón, la

28. Id.

29. Donaghy, Rori. 2015. «Falcon Eye: The Israeli-installed mass civil surveillance system of Abu Dhabi», Middle East Eye, 15-7-2015. Disponible en: https://cutt.ly/dmfrAzE.

30. Middle East Eye. 2020. «Israel and UAE held secret anti-Iran meeting at White House: Report (2020)», 4-2-2020. Disponible en: https://cutt.ly/8mfrHdz.

31. Dorsey, James. 2020. «Biden, Sanders, or Trump: US Policy Towards the Gulf Will Change Regardless", Inside Arabia, 11-3-2020. Disponible en: https://cutt.ly/JmfrCHm. 
aportación militar y tecnológica israelí resulta trascendental para garantizar un apoyo externo ante posibles amenazas. En contrapartida, Abu Dhabi está enviando señales preocupantes a los dirigentes palestinos, a pesar de que EAU niega cambio alguno en su rechazo a los asentamientos o la anexión de territorios palestinos ${ }^{32}$. Sin embargo, hay una disparidad evidente con respecto al periodo del emir Zayed, el cual, sin abrazar apasionadamente la reivindicación palestina, «se abstuvo de establecer contactos con el sionismo" ${ }^{33}$. En agosto de 2020, EAU consagró este cambio sustancial normalizando sus relaciones con Israel en un tratado que anuncia buscar un Oriente Medio «stable, peaceful, and prosperous» (U.S. Department of State, 2020). Aunque se ha señalado que la motivación principal es la postura común de ambos países frente a Irán ${ }^{34}$, el acuerdo prevé un volumen de comercio de cuatro billones de dólares en sectores que van desde la industria agroalimentaria hasta el turismo. La importancia concedida por EAU a una posible entente árabe-israelí ilustra, de nuevo, su tendencia a dirigir iniciativas propias que van más allá de sus entendimientos generales con Arabia Saudí. EUA, con su propio guión, intenta empujar a los aliados árabes - la Junta Militar en Sudán, por ejemplo- a proyectos de normalización con Israel. De hecho, el anuncio de Marruecos de establecimiento de relaciones plenas con aquel se debe, en parte, a gestiones a tres bandas de la diplomacia emiratí con Rabat, Washington y Tel Aviv, hasta el punto de ser calificada como el «gran patrón de la normalización árabe con Israel $\aleph^{35}$.

\section{CONCLUSIONES}

La política exterior de EAU tras la Primavera Árabe está condicionada por la menor presencia de EE. UU. en la zona, y busca extender su influencia en este escenario bajo la narrativa del antiislamismo. Para ello, ha optado por un espartanismo que favorece regímenes autoritarios y seculares en los países de Oriente Próximo, creando una red de influencia contraria al otro gran contendiente en este espacio, Catar, cuya estrategia se ha basado en la influencia a través del islamismo político. La participación de EAU en el bloqueo a Catar se debe leer en este contexto. La doctrina $M b Z$, fundamental sobre todo a partir de 2014, se centra en combatir el islamismo en

32. Al Quds al Arabi. 2020. "Al-Imarat tuakkid rafdaha li-mujattat israyiliyyin damm aradin filastiniyya", 10-5-2020. Disponible en: https://cutt.ly/GmftvIc.

33. Bakhakh, Yosra. 2018. "Mohamad bin Zayed, Zionist culprit in Mid-East», Tehran Times, 15-8-2018. Disponible en: https://cutt.ly/omftTdI.

34. Black, Ian. 2019. "Why Israel is quietly cosying up to Gulf monarchies», The Guardian, 19-32019. Disponible en: https://cutt.ly/3mdU6V6.

35. Hayy, Ibrahim. 2020. «Al Imarat batat al wakil al hasri li-l-l tatbi `fi al-mintaqa», Al Quds al Arabi, 26-12-2020. 
Oriente Medio, en especial a los HH.MM., para evitar cualquier contagio dentro de su ámbito regional.

Además, su implicación en el sur del Yemen muestra un interés creciente en puertos comerciales clave, como Adén, y la voluntad de expandirse por el Cuerno de África, como las bases militares establecidas allí demuestran. Estos movimientos sugieren, además, que EAU mantiene una postura propia no necesariamente alineada con la de aliados habituales como EE. UU. o Arabia Saudí. Por otro lado, la normalización de relaciones con Israel sugiere un cambio en las prioridades de EAU en Oriente Medio, donde la influencia económica y militar tiene más peso que la causa palestina.

Respecto a Irán, EAU ha mantenido una posición relativamente alineada con Arabia Saudí y EE. UU., que busca reducir su influencia en la zona. Esto no ha impedido, sin embargo, que mantenga relaciones notables comerciales con la república islámica y que haya sido uno de los principales mediadores en las tensiones con Washington. Para EAU, buscar una menor influencia de Irán no significa llegar a una guerra potencialmente dañina para ambos.

En suma, el process tracing de las actuaciones en política exterior de EAU demuestra que la llamada doctrina $M b Z$ se fundamenta en la batalla contra el islamismo, el espartanismo como método de intervención y la expansión en puertos comerciales clave en Oriente Medio y el Cuerno de África. Las prioridades locales y regionales de EAU, por tanto, le han llevado a tomar la iniciativa en el ámbito exterior, en una posición independiente de la de Arabia Saudí y convergente, o no, según el escenario y las prioridades de ambos estados. En este contexto, EAU ha optado por una vía espartana basada en un intervencionismo militar quirúrgico, convirtiéndose en uno de los principales actores en un nodo cada vez más importante en las relaciones de poder globales. Todo ello problematiza el esquema centro-periferia y refuerza el argumento de que el Golfo supone un nodo en sí mismo con un rol particular en el sistema-mundo actual, con una especial relevancia en Oriente Próximo, en el marco de la teoría de la dependencia de la que este artículo parte. A nivel de escala, EAU se ha convertido en uno de los actores fundamentales en el diseño del orden internacional y no solo en Oriente Medio.

\section{Referencias}

Adib-Moghaddam, Arshin. 2007. "Inventions of the Iran-Iraq war», Critical Middle Eastern Studies, 16 (1): 63-83. Disponible en: https://doi.org/10.1080/106699 20601148620.

África Fundación Sur. 2020. "Concesión a una empresa de Dubai de la terminal del puerto namibio de Walvis Bay», Noticias, 11-3-2020. Disponible en: http://www. africafundacion.org/spip.php?article36569.

African Intelligence. 2015. «How Eritrea benefits from the diplomatic crisis between Djibouti and the UAE», TesfaNews, 15-5-2015. Disponible en: https:/cutt.ly/ VmdgMkf. 
Al-Otayba, Youssef. 2014. "The Moderate Middle East must act», Embassy of the United Arab Emirates, News Media, 9-9-2014. Disponible en: https://cutt.ly/0mdhotL.

Amin, Samir. 1974. "Accumulation and Development: A Theoretical Model», Review of African Political Economy, 1: 9-26. Disponible en: https://doi.org/10.1080/ 03056247408703234.

Ayoob, Mohammed. 2002. «Inequality and Theorizing in International Relations: The Case for Subaltern Realism», International Studies Review, 4 (3): 27-48. Disponible en: https://doi.org/10.1111/1521-9488.00263.

Badi, Emadeddin. 2020. "Russia isn't the only one getting its hands dirty in Libya», Foreign Policy, 21-4-2020. Disponible en: https://cutt.ly/3mdhvgt.

Barany, Zoltan. 2016. The Bahrain Defense Force: the monarchy's second-to-last line of defense. Center for Strategic and International Studies. Disponible en: https://cutt. ly/rmdjngD.

Bassiouni, Mahmoud. C. et al. 2011. Report of the Bahrain Independent Commission of Inquiry. Disponible en: http://files.bici.org.bh/BICIreportEN.pdf.

Brehony, Noel. 2020. "The UAE's role in the Yemen crisis", en Stephen W. Day y Noel Brehony (eds.), Global, Regional, and Local Dynamics in the Yemen Crisis. Nueva York: Palgrave Macmillan. Disponible en: https://doi.org/10.1007/978-3030-35578-4_9.

Collier, David. 2011. «Understanding process-tracing», Political Science and Politics, 44: 823-830. Disponible en: https://doi.org/10.1017/S1049096511001429.

Davidson, Christopher. 2008. Dubai: the vulnerability of success. Oxford: Oxford University Press.

Davidson, Christopher. 2013. After the Sheikhs: the coming collapse of the Gulf monarchies. Londres: Hurst.

Elshestawy, Yasser. 2009. Dubai: behind an urban spectacle. Londres: Routledge. Disponible en: https://doi.org/10.4324/9780203869703.

Freer, Courtney. 2018. Rentier Islamism. the influence of the Muslim Brotherhood in Gulf Monarchies. Oxford: Oxford University Press. Disponible en: https://doi. org/10.1093/oso/9780190861995.001.0001.

Fulton, Jonathan. 2020. China's Relations with the Gulf Monarchies. Londres: Routledge.

Goertz, Gary y James Mahoney. 2012. A Tale of Two Cultures: Qualitative and Quantitative Research in the Social Sciences. Princeton: Princeton University Press.

Hanieh, Adam. 2019. Money, markets, and monarchies: The Gulf Cooperation Council and the political economy of the contemporary Middle East. Cambridge: Cambridge University Press. Disponible en: https://doi.org/10.1017/9781108614443.

Hellyer, Peter. 2001. "The Evolution of UAE Foreing Policy», en Ibrahim Al Abed y Peter Hellyer (eds.), United Arab Emirates. A new perspective. Londres: Trident Press.

Hey, Jeanne A. K. 1993. «Foreign Policy Options under Dependence: A Theoretical Evaluation with Evidence from Ecuador", Journal of Latin American Studies, 25 (3): 543-574. Disponible en: https://doi.org/10.1017/S0022216X00006660. 
Jones, Calvert. 2017. Bedouins into bourgeois. Remaking citizens for Globalization. Cambridge: Cambridge University Press. Disponible en: https://doi.org/10.1017/ 9781316800010.

Kazzi, Habib. 2017. "Is the Gulf Cooperation Council (GCC) customs union a myth?», Asian Journal of Business and Management, 5 (5): 150-156.

Krieg, Andreas. 2019. "The Weaponization of Narratives Amid the Gulf Crisis», en Andreas Krieg (ed.), Divided Gulf. The Anatomy of a Crisis. Londres: Palgrave Macmillan. Disponible en: https://doi.org/10.1007/978-981-13-6314-6.

Lynch, Marc. 2015. "Obama and the Middle East. Rightsizing the U.S. Role», Foreign Affairs (septiembre/octubre). Disponible en: https://cutt.ly/OmdkKQ2.

Matthiesen, Toby. 2013. Sectarian Gulf. Bahrain, Saudi Arabia, and the Arab Spring that wasn't. Stanford: Stanford University Press. Disponible en: https://doi. org/10.1515/9780804787222.

Mello, Alex y Michael Knights. 2016. "West of Suez for the United Arab Emirates», War on the Rocks, 2-9-2016. Disponible en: https://cutt.ly/QmfepDa.

Nye, Joseph. 2012. The future of power. Nueva York: Public Affairs.

Owen, John M. 1994. "How Liberalism Produces Democratic Peace», International Security, 19 (2): 87-125. Disponible en: https://doi.org/10.2307/2539197.

Pike, David. 1994. "Cross border hydrocarbon reserves», en Richard N. Schofiled, (ed.), Territorial foundations of the Gulf States. Londres: UCL Press.

Roberts, David. 2014. "Qatar and the Muslim Brotherhood: Pragmatism or Preference?», Middle East Policy, 21 (3): 84-94. Disponible en: https://doi.org/10.1111/ mepo.12084.

Sadeq Shurab, N. 1987. Al-siyasa al-jariyiyya li Dawlat al-Imarat al-'Arabiyya al-Muttahida. Al Ain: Dar al-Kitaba al-Yami'i.

Salame, Ghassan. 1981. "Ta'thir al-alaqaat al-'arabiyya wa al-dawliyya», en Actas del Congreso Internacional sobre las experiencias unionistas en el mundo árabe. La experiencia de los Emiratos Árabes Unidos, Abu Dhabi, octubre de 1981. Beirut: Markaz Dirasat al-Wahda al-Arabiyya.

The Soufian Center. 2020. "Instability in Southern Yemen exposes rift between Riyadh and Abu Dhabi», IntelBrief, 6-5-2020. Disponible en: https:/cutt.ly/ fmdlqUx.

U. S. Department of State. 2020. Abraham Accords Peace Agreement: Treaty of Peace, Diplomatic Relations, and Full Normalization Between the United Arab Emirates and the State of Israel. Disponible en: https://cutt.ly/Amdlpm7.

Wallerstein, Immanuel. 2004. World-Systems Analysis: An Introduction. Durham: Duke University Press. Disponible en: https://doi.org/10.2307/j.ctv11smzx1.

Zuboff, Shoshana. 2019. The age of surveillance capitalism: the fight for a human future at the new frontier of power. Nueva York: Public Affairs.

Presentado para evaluación: 20 de octubre de 2020 .

Aceptado para publicación: 2 de junio de 2021. 


\section{JAVIER GUIRADO \\ jguirado1@gsu.edu}

Es estudiante de doctorado en la Georgia State University. Su investigación explora el papel de los movimientos sociales y las élites en Catar durante el siglo xx y su relación con movimientos anticoloniales en el sur global. Ha sido investigador invitado en el Nederlands-Vlaams Instituut en El Cairo y es máster en Estudios Árabes e Islámicos Contemporáneos por la Universidad Autónoma de Madrid.

\section{IGNACIO GUTIÉRREZ DE TERÁN}

ignaciog.deteran@uam.es

Es profesor titular del Departamento de Estudios Árabes e Islámicos de la Universidad Autónoma de Madrid. Especializado en procesos de transición democrática en Oriente Medio, ha publicado numerosos artículos y libros sobre la situación política actual en numerosos países árabes y las movilizaciones populares que han tenido lugar en ellos. Cabe destacar su monografía Las revoluciones árabes (2017). 